\title{
Contribución al estudio del proceso de gelación de la pectina del níspero de la sierra
}

\author{
Nancy Chasquibol Silva, Juan Carlos Morales Gomero \\ Universidad de Lima. \\ Ingeniería Industrial n. 28, 2010, ISSN 1025-9929, pp. 157-176 \\ Recibido: 28 de setiembre del 2009 / Aprobado: 28 de octubre del 2009
}

RESUMEN: El mecanismo de gelación de la pectina depende del grado de metoxilación. Las pectinas con alto metoxilo forman geles debido a las interacciones hidrofóbicas y al enlace del puente de hidrógeno entre las moléculas de la pectina. Los resultados de esta investigación indican que el $\mathrm{NaOH}$ añadido a las soluciones de pectina de alto metoxilo del níspero de la sierra (Mespilus sp.) forma geles en una relación directa con la concentración. La disminución del $\mathrm{pH}$ de las soluciones de pectina de este fruto causó el incremento de la fuerza del gel a pH cercano a 3,6. Las soluciones de pectina del níspero de la sierra formaron geles con $\mathrm{NaCl}$ en concentraciones entre 0,2 y $0,8 \mathrm{M}$. La adición de una pequeña cantidad de $\mathrm{Ca}^{+2}$ promovió la gelación de la pectina del fruto. La fuerza del gel se incrementó también con la adición de sacarosa.

Palabras clave: pectinas / gelación / espectroscopia UV / viscosidad / nísperos

\section{Contribution to the study of gelation process of "níspero de la sierra" pectin}

ABSTRACT: The mechanism of pectin gelation depends of the degree of methoxylation. High methoxyl pectin gels due to hydrophobic interactions and hydrogen bonding between pectin molecules. The results of this research indicate that $\mathrm{NaOH}$ added to high methoxyl pectins solutions of "níspero de la sierra" (Mespilus sp.) leads to gel formation in a concentration-depended manner. Decreasing pHs of "níspero de la sierra" pectin solutions caused increases en gel strength between $\mathrm{pH}$ 3,6 níspero de la sierra pectin solutions formed gels at $\mathrm{NaCl}$ concentration between 0,2 y $0,8 \mathrm{M}$. The addition of small amount of $\mathrm{Ca}^{+2}$ promoted the gelation of "níspero de la sierra" pectin. The gel strength of the "níspero de la sierra" gels was further enhanced by sucrose.

Keywords: pectins / gelation / UV spectroscopy / viscosity / nísperos 


\section{INTRODUCCIÓN}

El níspero de la sierra (Mespilus sp.) es un árbol que crece en estado silvestre en los departamentos de Ayacucho y Junín. El fruto se caracteriza por tener una cubierta membranácea amarillo-naranja que envuelve una pulpa dura y dulce, un poco ácida y muy agradable al paladar. La cosecha del fruto se realiza en los meses de abril a setiembre, y entre sus usos se encuentra la preparación de dulces y mermeladas.

En investigaciones anteriores (Chasquibol et al. 2008: 175-199), se ha encontrado que la pulpa del fruto del níspero de la sierra destaca por su contenido de carbohidratos (16,66\%), fibra $(9,12 \%)$, grasas $(0,78 \%)$ y proteínas $(0,51 \%)$. Por el procedimiento de extracción ácida, se ha encontrado que la pulpa del níspero de la sierra presenta un rendimiento máximo de pectina de alto metoxilo de $23,85 \%$. Dichas pectinas se caracterizan por su contenido de humedad (17,80\%), cenizas $(3,47 \%)$, metoxilos $(14,55 \%)$, ácido anhidro galacturónico $(87,93 \%)$, grado de metoxilación $(89,15 \%)$, grado de esterificación $(86,24 \%)$, grado de gelificación $(200)$, viscosidad relativa $(1,49)$, viscosidad intrínseca $(4,25)$ y peso molecular $(10183,5)$. Las características fisicoquímicas y el análisis espectrofotométrico infrarrojo confirmaron que las pectinas extraídas de la pulpa del níspero de la sierra son de alto metoxilo, alta pureza y buena calidad.

Las propiedades fisicoquímicas de las pectinas relacionadas con la función que desarrollan como fibra y como agente espesante en los alimentos, están determinados en gran medida por el grado de metoxilación de los grupos carboxílicos (Barford et al. 1986: 2576-2578). Se enontró que el grado de metoxilación en las muestras analizadas están por encima del 50\% (Bernhard et al. 2004: 375-378), lo cual confirma que las pectinas de la pulpa del níspero de la sierra son de gelificación ultrarrápida (rapid set). Por lo tanto, las pectinas estudiadas pueden ser empleadas en el procesamiento de mermeladas, confituras y jaleas, debido a la facilidad con la que se gelifican. Los mecanismos de la gelificación de las pectinas dependen del grado de metoxilación, ello debido a interacciones hidrofóbicas y a los puentes de hidrógeno entre las moléculas de la pectina especialmente a bajo $\mathrm{pH}(\approx 3)$ y con alto contenido de azúcar (Oakenfull 1991: 87-108). 
Figura 1

Mecanismo de gelación de pectinas con alto metoxilo (HM) (interacciones hidrofóbicas y puentes de hidrógeno)

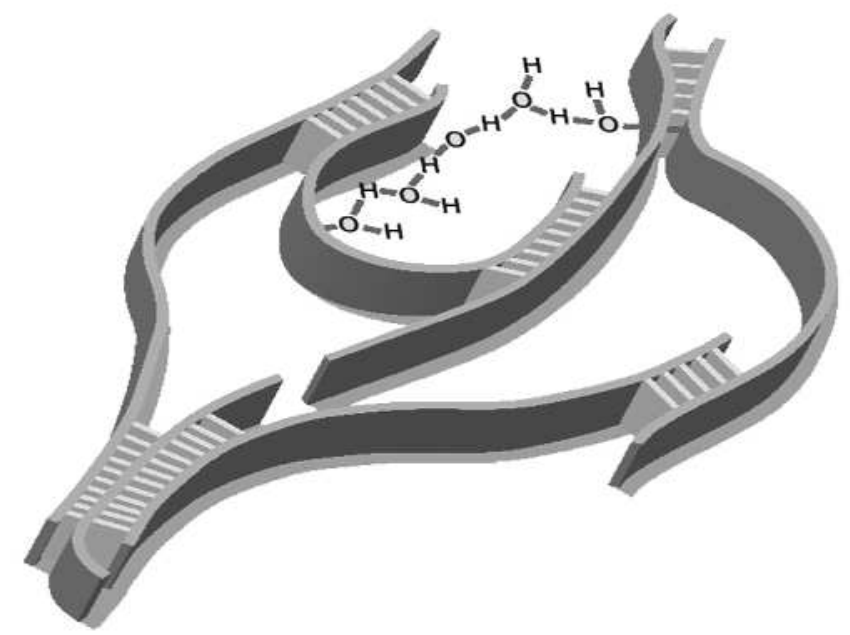

Fuente: <http://www.herbstreith-fox.de/en/informative-literature/informative-literaturefrom-rd-and-tech-application.html>.

Por el contrario, las pectinas de bajo metoxilo forman geles en presencia de calcio $\mathrm{u}$ otros cationes divalentes en un amplio rango de $\mathrm{pH}$ con o sin azúcar. El modelo de la "caja de huevos" ha sido ampliamente aceptado para la formación de geles de pectato de calcio (Grant et al. 1973: 195-198) y describe que la gelación de las pectinas de bajo metoxilo ocurre como consecuencia de la interacción entre los iones de calcio y los grupos carboxilos de las cadenas de las pectinas (Axelos y Thibault 1991: 109-118) (figura 2). Debido a su bajo contenido de azúcar, las pectinas de bajo metoxilo tienen muchas aplicaciones en alimentos bajos en calorías y en alimentos dietéticos (May 1990: 79-99).

Las pectinas son ampliamente usadas en las industrias alimentaria, cosmética y farmacéutica, como estabilizante y como agente quelante (Koseki et al. 1986: 1329-1332). Las pectinas son empleadas, además, como agentes estabilizantes en la industria láctea, donde la principal función es actuar como un hidrocoloide protector junto con la caseína a bajo pH (Gregory 1986: 211-240). 
Figura 2

Mecanismo de gelación de pectinas con bajo metoxilo (complejación de iones calcio). Modelo de la "caja de huevos"

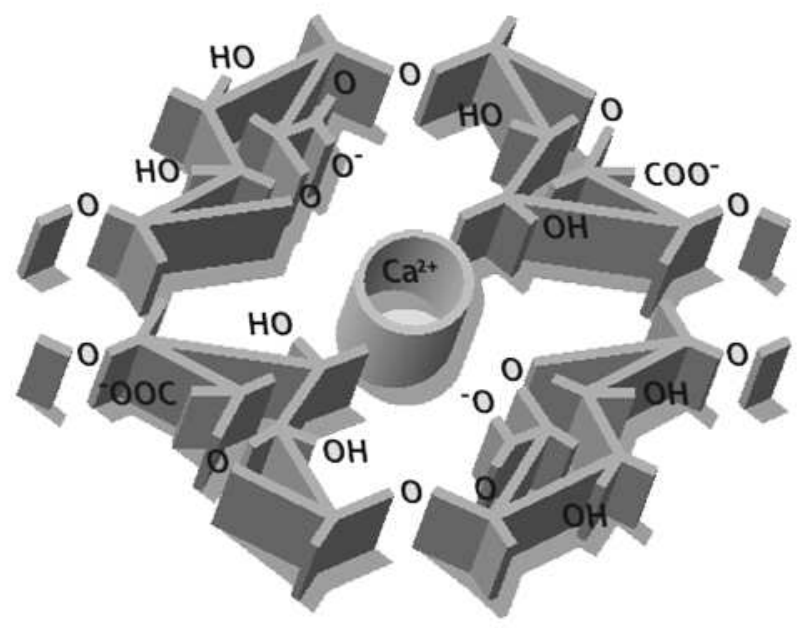

Fuente: <http://www.herbstreith-fox.de/en/informative-literature/informative-literaturefrom-rd-and-tech-application.html>.

Una de las propiedades más importantes de las pectinas es la viscosidad, que se ve afectada por el peso molecular, el grado de metilación, pH y la presencia de contraiones (Phatak et al. 1988: 830-833). El comportamiento de la viscosidad cambia también con la concentración y la temperatura. La gelación está relacionada con la viscosidad de las soluciones de la pectina.

Durante el procesamiento, almacenamiento, comercialización y consumo, los alimentos fluidos presentan diferentes concentraciones y están sujetos a diversas temperaturas. La información de la viscosidad y el conocimiento del comportamiento reológico de zumos y pulpas de frutas son fundamentales para la ingeniería de procesos y es necesario describirlos para el diseño de tuberías, selección de bombas, diseño y operación de intercambiadores de calor, sistemas de agitación, mezclas de productos y selección de envases.

Es necesario conocer el efecto de la temperatura y la concentración sobre el comportamiento reológico para un buen entendimiento y dimensionamiento de operaciones unitarias, como el tratamiento tér- 
mico, el congelamiento y la concentración (Barbosa-Canovas et al. 1993: 39-49).

La inexistencia de datos reológicos en la literatura de frutas tropicales ha llevado a la industria a asumir, en el proceso de fabricación de estos zumos, condiciones semejantes a las aplicadas en la producción del zumo de naranja (Vidal et al. 2000: 70-76).

La reología es la ciencia del flujo que estudia la deformación de un cuerpo sometido a esfuerzos extremos. Su estudio es esencial en muchas industrias, incluyendo la de plásticos, pinturas, alimentos, aceites, detergentes, fármacos, cosméticos, lubricantes, entre otros.

Algunos fluidos alimenticios como las pastas de frutas y otros vegetales, el ketchup, la mostaza y papillas para bebés son fluidos no newtonianos y presentan comportamiento tixotrópico de difícil modelamiento, pues necesitan parámetros que cuantifiquen su dependencia con el tiempo de cizallamiento (Rao 1986: 398; Choi y Yoo 2004: 801-805).

De manera general, y sin considerar los efectos temporales, el modelo de Herschel-Bulkley, que se indica en la ecuación (1), puede ser usado para describir el comportamiento de diversos tipos de fluidos, pues engloba todos los modelos hasta hoy conocidos (Rao 1986).

$$
\tau=\tau_{0}+K(\gamma)^{n}
$$

donde:

$\tau=$ tensión de cizallamiento (Pa.s)

$\gamma=$ tasa de deformación $\left(s^{-1}\right)$

$\tau_{0}=$ tensión residual $(P a)$

$K=$ índice de consistencia $(P a . s)$

$\mathrm{n}=$ índice de comportamiento en el flujo

Los fluidos alimenticios que no contienen fibras en suspensión generalmente se ajustan a un caso especial de esa expresión, en la cual la tensión residual $\left(\tau_{0}\right)$ es nula, o se asume como cero, de forma que la ecuación (1) se transforma en: 


$$
\tau=K_{M}(\gamma)^{n}
$$

La ecuación (2) es conocida como la Ley de Potencia o Power-law. La mayor parte de los alimentos muestran comportamiento pseudoplástico $(\mathrm{n}<1,0)$, o sea, la viscosidad aparente disminuye a medida que la tasa de deformación y el tiempo de cizallamiento aumentan, debido a la orientación de las moléculas en la dirección del flujo y la quiebra de agregados, que tornan la resistencia al movimiento cada vez menor (Rao 1986).

En la revisión bibliográfica se encontró que la pulpa de camu-camu (Myrciaria dubia) presenta un comportamiento pseudoplástico, según el modelo de la Ley de la Potencia. Además, se encontró que la viscosidad aparente de su pulpa disminuye con el aumento de la temperatura (Arévalo et al. 2006). Por otro lado, se estudiaron las propiedades reológicas de dos polisacáridos pépticos presentes en la oppuntia spp., encontrándose buenas propiedades de gelación en presencia de $\mathrm{CaCl}_{2}$ por un mecanismo de cooperación de $\mathrm{Ca}^{2+}$ "caja-huevos" (Goycoolea et al. 2003: 17-29).

Una nueva pectina fue extraída de las hojas de Krueo Ma Noy (Cissanpelos pareira), una planta silvestre que crece en Tailandia. La gelación fue observada cuando la pectina de las hojas de Krueo Ma Noy no excedió la concentración de $0,5 \%(\mathrm{p} / \mathrm{v})$. La adición de $\mathrm{NaCl}$ incrementó significativamente la fuerza del gel de la pectina a concentraciones de la sal por debajo de $0,4 \mathrm{M}$. La pectina de las hojas de Krueo Ma Noy también es sensible a la adición de iones $\mathrm{Ca}^{2+}$. La adición de $\mathrm{CaCl}_{2} 1 \mathrm{mM}$ disminuyó significativamente la fuerza de gel y la excesiva cantidad de $\mathrm{Ca}^{2+}(>3 \mathrm{mM})$ provocó la precipitación de la pectina, posiblemente debido a las propiedades enlazantes del calcio con el grupo carboxilo. Además, se observó que la adición de azúcar aumenta la fuerza del gel (Singthong et al. 2005: 793-801).

Los componentes gelificantes de la solución de la pectina del fruto del níspero de la sierra podrían ser otra fuente natural de polisacáridos. Sin embargo, no existe información científica respecto de las propiedades reológicas de este fruto. Este artículo es el primero en contribuir al estudio del uso potencial de este nuevo biopolímero, analizando las propiedades reológicas de la solución de la pectina del fruto del níspero de la sierra, mediante el viscosímetro y por espectrofotometría ultravioleta-visible. 


\section{MATERIALES Y MÉTODOS}

\subsection{Materia prima}

La pectina del fruto del níspero de la sierra con porcentaje de metoxilos (14,55\%), ácido anhidro-galacturónico $(87,93 \%)$, grado de metoxilación $(89,15 \%)$, grado de esterificación $(86,24 \%)$ obtenida por sucesivas extracciones, según el proceso descrito por Chasquibol, Arroyo y Morales (2007: 28-34), fue empleada para el estudio de las propiedades reológicas de la solución de la pectina del fruto del níspero de la sierra, mediante el viscosímetro y por espectrofotometría ultravioleta-visible.

\subsection{Reactivos y equipos utilizados}

Reactivos:

- Acido clorhídrico, grado reactivo, J. T. Baker

- Cloruro de sodio, grado reactivo Merck

- Cloruro de calcio, grado reactivo Merck

- Hidróxido de sodio, grado reactivo Merck

- Sacarosa, grado reactivo Merck

- Patrón de pectina de manzana USP testing Sigma, P84471-100G

- Patrón de pectina de cáscara de limón con ácido galacturónico $>=74 \%$ (base anhidra) Sigma P91135-100G

Equipos:

- Espectrofotómetro FTIR, Nicolet Modelo 410

- Espectrofotómetro UV-V, Perkin Elmer, Lamda 40 de doble haz

- Espectrofotómetro AA, Shimatzu, versión AA-6800

- Determinador de gravedad específica Fisher-Scientific MettlerToledo Instrument/DA- 110M

- Viscosímetro Ostwald 20-75 sec

- Balanza analítica OHAUS/AP $210 \mathrm{~S}$

- Estufa Memmert modelo ULP 500

- Potenciómetro Termo Orion

- Triturador de alimentos, GRINDUMIX GM 100-RETSCH, speed 5000, $1000 \mathrm{rpm}$ 
- Plancha de calentamiento, THERMOLYNE + MIRAK TM

- Bomba de vacío

\section{PARTE EXPERIMENTAL}

En la pectina seca del fruto del níspero de la sierra se analizó por absorción atómica, el contenido de sodio, potasio y calcio. Se determinaron los espectros infrarrojos de los patrones de las pectinas de manzana y del limón para determinar el grado de esterificación y para la identificación de pectinas de alto y bajo metoxilo (Manrique et al. 2002: 99-107).

Las muestras de pectina seca del fruto del níspero de la sierra fueron empleadas a diferentes concentraciones, para el estudio viscosimétrico y para el análisis espectrofotométrico. El estudio viscosimétrico de la gelación y las propiedades reológicas fueron determinados con un viscosímetro Ostwald-Cannon-Fenske tipo capilar a temperatura ambiente (Singthong et al. 2005: 93-801).

El análisis espectrofotométrico del tiempo de gelación fue realizado a $278 \mathrm{~nm}$ en modo de transmitancia (\%), usando cubetas con $1 \mathrm{~cm}$ de paso óptico.

Todas las soluciones para el análisis espectrofotométrico estuvieron a la temperatura ambiente $\left(25^{\circ} \mathrm{C}\right)$ para asegurar resultados confiables.

El tiempo en el cual el máximo cambio en la transmitancia ocurrió fue tomado como el tiempo de inicio en la formación del gel de la pectina (Bernhard et al. 2004: 375-378).

\section{RESULTADOS Y DISCUSIÓN}

\subsection{Composición química}

En la tabla 1 se muestra el porcentaje de sodio, potasio y calcio presente en la pectina del fruto del níspero de la sierra, determinado por espectrofotometría por absorción atómica a la llama. 
Tabla 1

Análisis de minerales en la pectina del fruto del níspero de la sierra

\begin{tabular}{|l|c|}
\hline Parámetro & Composición (\%) \\
\hline Sodio & 0,40 \\
Potasio & 1,83 \\
Calcio & 0,94 \\
\hline
\end{tabular}

\subsection{Espectrofotometría infrarroja}

Los espectros infrarrojos de los patrones de las pectinas de la manzana y el limón se indican en las gráficas 1 y 2 , respectivamente. Estos espectros tienen características similares, ya que presentaron un alargamiento pronunciado entre $1.626,15$ y $1.646,99 \mathrm{~cm}^{-1}$, que corresponde a la banda de vibración de tensión simétrica del grupo $\mathrm{COO}^{-}$, mientras que las bandas que aparecen entre $1.746,2 \mathrm{~cm}^{-1}$ y $1.749,19 \mathrm{~cm}^{-1}$ corresponden a los grupos carbonilos de los grupos $\mathrm{COOH}$ y de los grupos acetilos $\left(\mathrm{COOCH}_{3}\right)$ de la pectina.

\section{Gráfica 1}

Espectro infrarrojo del patrón de la pectina de manzana

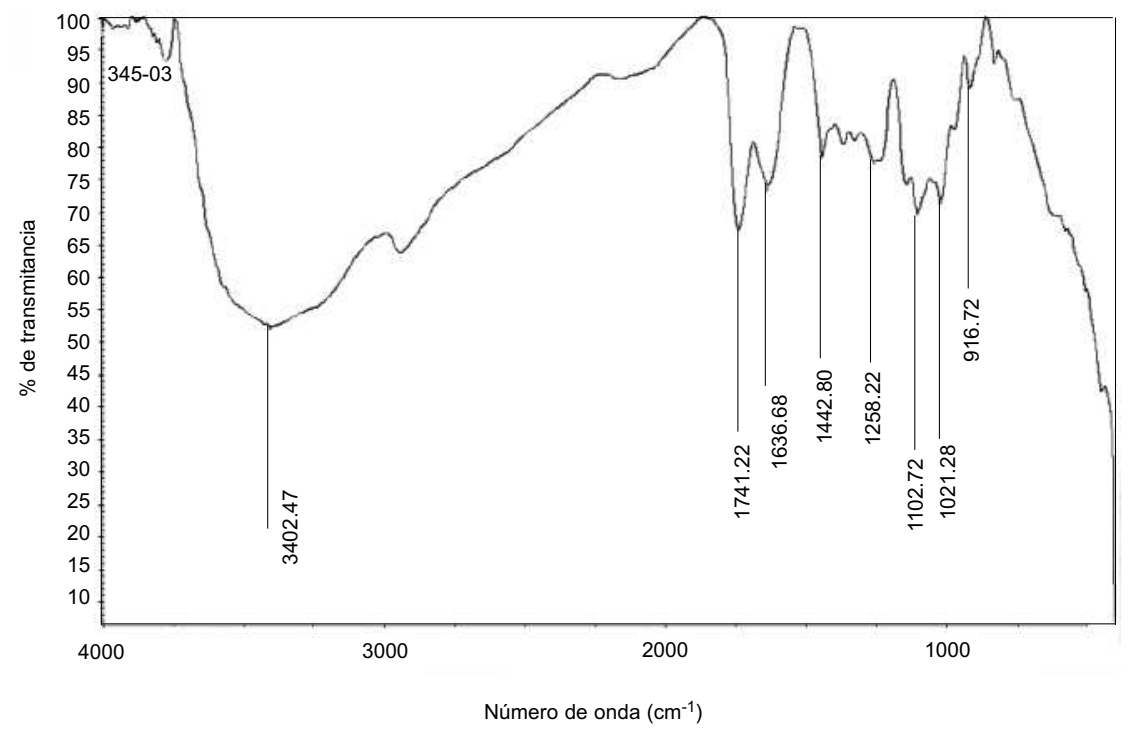


La presencia de ambas bandas confirma el alto grado de esterificación y la presencia de pectina de alto metoxilo en ambos patrones.

\section{Gráfica 2}

Espectro infrarrojo del patrón de la pectina de limón

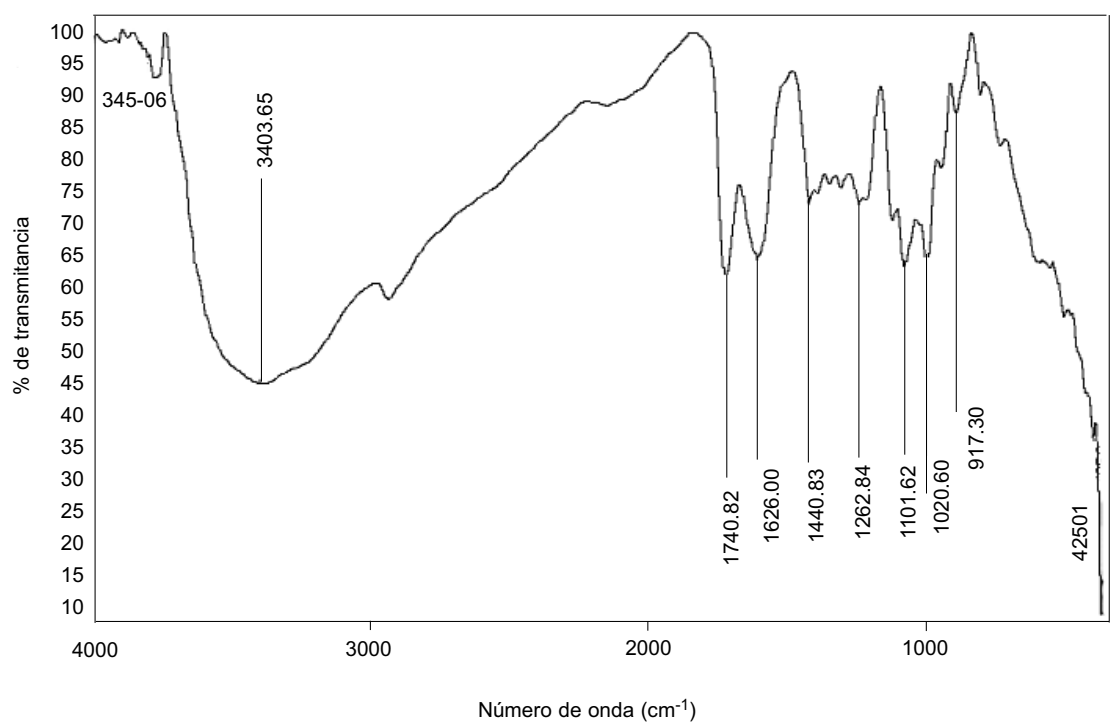

\subsection{Propiedades reológicas}

\subsubsection{Estudio espectrofotométrico de la gelación de la pectina}

Los espectros ultravioleta fueron registrados para muestras de pectinas disueltas en agua desionizada a una concentración $15 \mathrm{mg} / \mathrm{mL}$. En la gráfica 3 se observa que el máximo de absorbancia de la muestra de pectina del níspero de la sierra coincide con el máximo de absorbancia del patrón de la pectina del limón.

Se realizó el estudio de la formación de los geles de la pectina del níspero de la sierra inducidos por la presencia de $\mathrm{NaOH}$, para una concentración de pectina de $3,3 \mathrm{mg} / \mathrm{mL}$. El tiempo de gelación fue tomado inmediatamente después de adicionar el $\mathrm{NaOH} 0,33 \mathrm{M}$, conteniendo $\mathrm{NaCl}$ en concentraciones de $50 \mathrm{mM}, 90 \mathrm{mM}$ y $120 \mathrm{mM}$, respectivamente. 


\section{Gráfica 3}

Espectro ultravioleta de la solución de la pectina del níspero de la sierra y del patrón de la pectina del limón

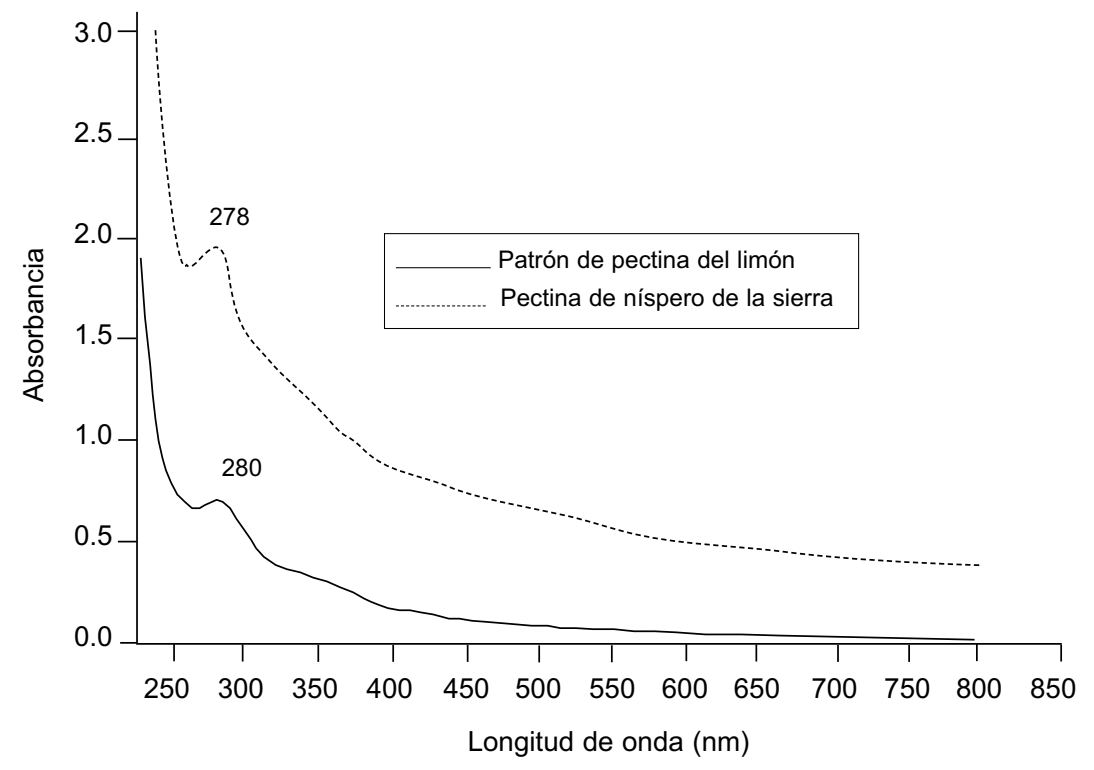

Gráfica 4

Porcentaje (\%) de transmitancia de la solución de la pectina del níspero de la sierra

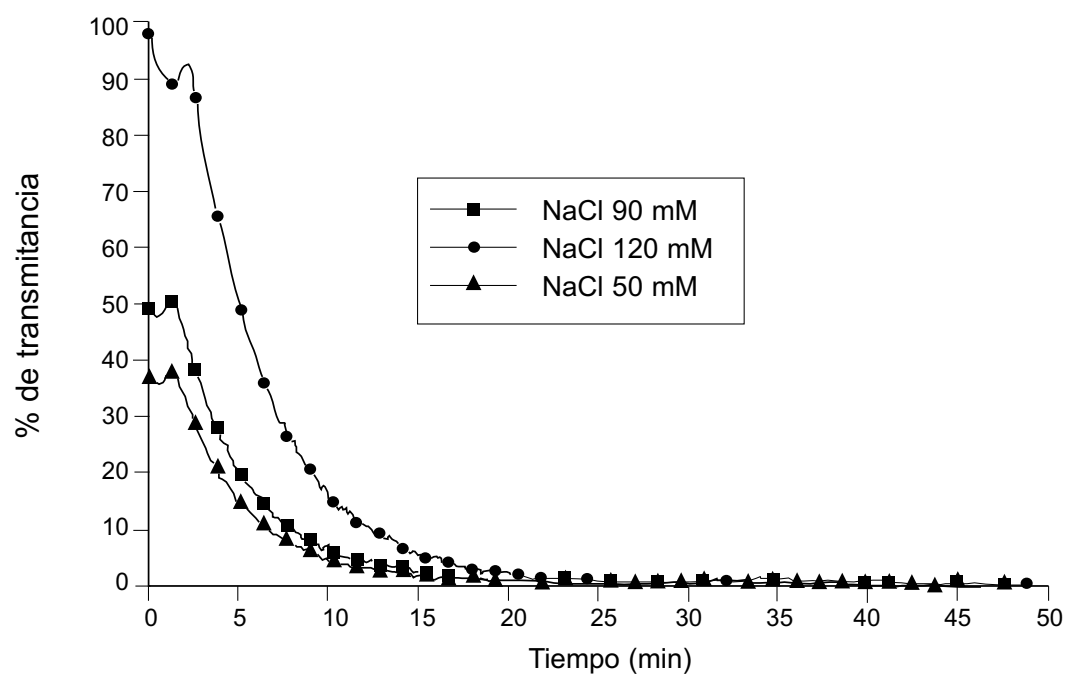


En la gráfica 4 se puede observar que para las diferentes concentraciones de $\mathrm{NaCl}$, en todos ellos el gel comienza a formarse a los dos minutos de adicionado el $\mathrm{NaOH}$, siendo más rápida la formación del gel a una concentración de $50 \mathrm{mM}$ de $\mathrm{NaCl}$.

\subsubsection{Comportamiento del flujo}

\subsubsection{Efecto de la concentración}

La tabla 2 y la gráfica 5 muestran los resultados obtenidos para la viscosidad relativa de la solución de la pectina del níspero de la sierra a concentraciones de $0,1-2,5 \%(\mathrm{p} / \mathrm{v})$.

Tabla 2

Viscosidad relativa de la solución de la pectina del níspero de la sierra a concentraciones de $0,1-2,5 \%(p / v)$

\begin{tabular}{cccc}
\hline Muestra & Concentración de pectina $(\% \mathbf{p} / \mathbf{v})$ & Tiempo (segundos) & $\boldsymbol{\eta}_{\text {relativa }}$ \\
\hline 1 & 0,10 & 109 & 1,47 \\
2 & 0,25 & 150 & 1,58 \\
3 & 0,50 & 166 & 2,06 \\
4 & 1,00 & 229 & 2,90 \\
5 & 1,50 & 599 & 7,97 \\
6 & 2,00 & 1857 & 25,40 \\
7 & 2,50 & 1914 & 26,21 \\
\hline
\end{tabular}

En la gráfica 5 se observa la dependencia de la viscosidad relativa con respecto al aumento de la concentración, se demuestra que la viscosidad es baja a concentraciones menores $(0,1-0,25 \%)$ y el flujo presenta características de tipo no-newtoniano (Singthong et al. 2005: 793-801).

A concentraciones intermedias (0,5-1\%), aumentan las interacciones entre las moléculas de la pectina, lo cual conduce a un aumento en la viscosidad y el flujo de la solución de pectina presenta un comportamiento de tipo newtoniano.

A concentraciones altas de pectina (1,5-2,5\%), se forman enlaces por interacciones físicas, dando lugar a la formación de geles y aumentando la viscosidad de la pectina. 


\section{Gráfica 5}

Viscosidad relativa de la solución de la pectina del níspero de la sierra a concentraciones de $0,1-2,5 \%(p / v)$

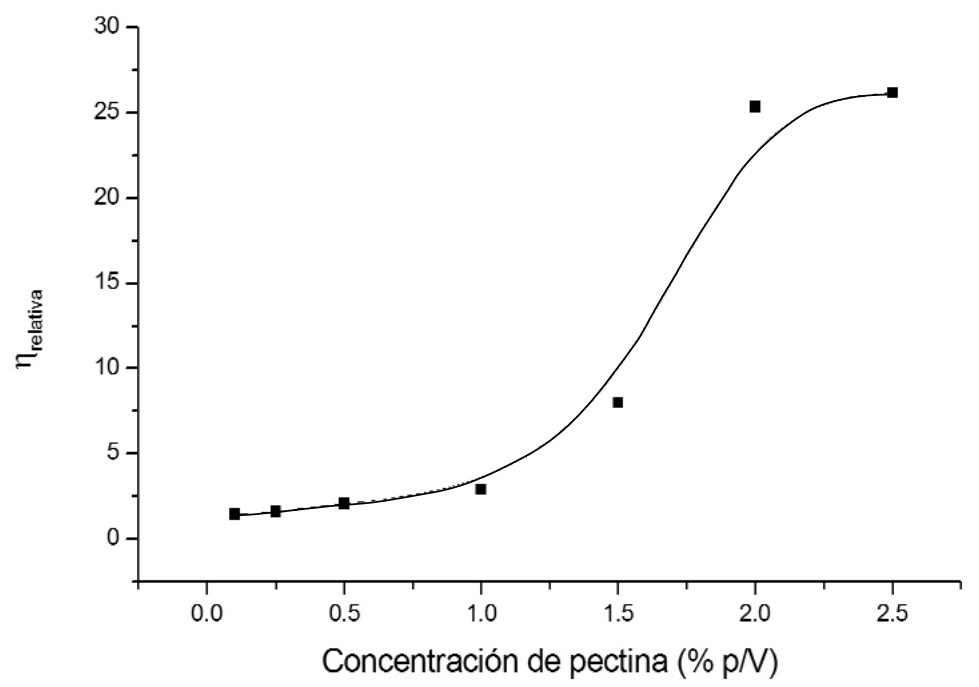

\subsubsection{Comportamiento viscoelástico}

\subsubsection{Efecto del $\mathrm{pH}$}

La tabla 3 y la gráfica 6 muestran los resultados obtenidos de la dependencia experimental encontrada para la viscosidad relativa de la solución de la pectina del níspero de la sierra al 0,5\% con el $\mathrm{pH}$ de la solución.

Tabla 3

Resultados de la viscosidad relativa de la pectina del níspero de la sierra al $0,5 \%$ con el $\mathrm{pH}$

\begin{tabular}{cccc}
\hline Muestra & pH & Tiempo (segundos) & $\boldsymbol{\eta}_{\text {relativa }}$ \\
\hline 1 & 2 & 154 & 1,94 \\
2 & 4 & 183 & 2,93 \\
3 & 5 & 142 & 1,84 \\
4 & 6 & 151 & 2,75 \\
5 & 7 & 216 & 2,79 \\
6 & 8 & 230 & 2,91 \\
7 & 10 & 198 & 2,64 \\
\hline
\end{tabular}




\section{Gráfica 6}

Dependencia de la viscosidad relativa de la solución de la pectina del níspero de la sierra al $0,5 \%$ con el $\mathrm{pH}$ del medio

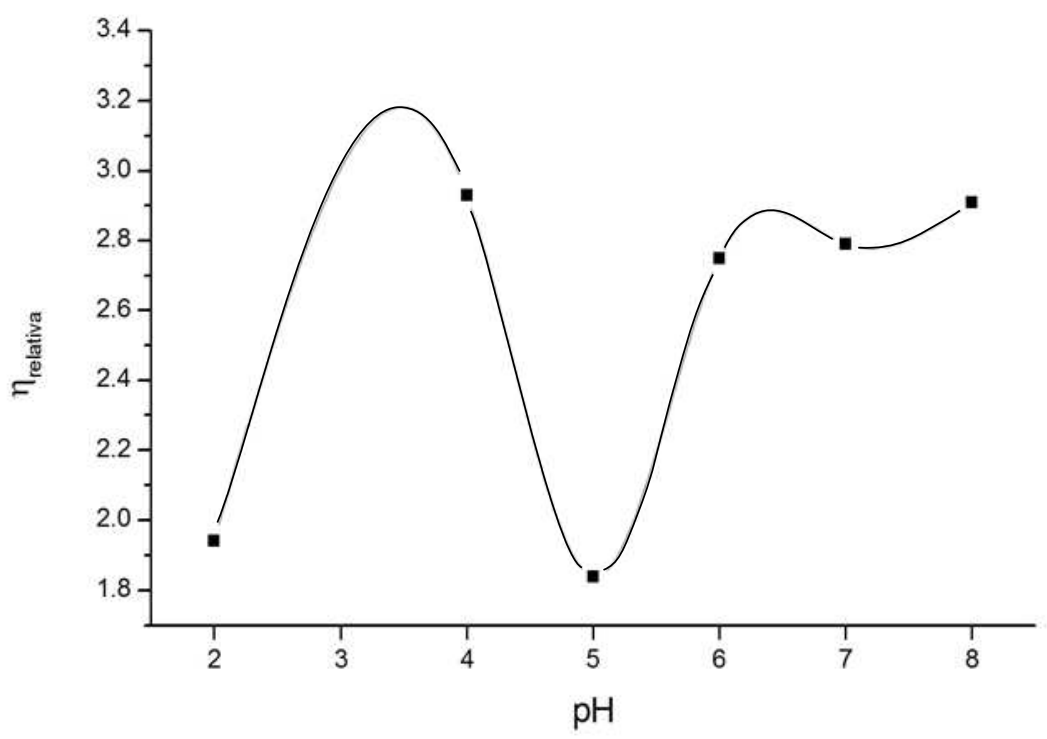

En la gráfica 6 se observa que la viscosidad más baja corresponde al punto isoeléctrico ( $\mathrm{pH}$ 5), en el cual la molécula se encuentra enrollada y debido al pequeño volumen que ocupa la molécula enrollada, la viscosidad del líquido es mínima. Cuando el pH disminuye o se incrementa, la viscosidad aumenta, debido a que la molécula de la pectina se desarrolla progresivamente y la viscosidad aumenta hasta alcanzar un máximo (Voyutskys 1978: 510-511).

Las pectinas de alto metoxilo forman geles si el $\mathrm{pH}$ es bajo, cercano a 3,6 (Oakenful y Scout 1984: 1093-1098); de acuerdo con la gráfica 6 , la solución de la pectina de alto metoxilo del níspero de la sierra forma geles también a $\mathrm{pH}$ cercano a 3,6.

\subsubsection{Efecto de los co-solutos}

El efecto de la fuerza iónica y el tipo de ión que influye en el comportamiento viscoelástico es importante para determinar si la muestra se comporta como un polielectrolito, y también para evaluar las propiedades funcionales reológicas (Medina et al. 2000: 417-424). 
La tabla 4 y la gráfica 7 muestran los resultados obtenidos al añadir $\mathrm{NaCl}$ a la solución de la pectina del níspero de la sierra al 0,5\%.

Tabla 4

Efecto del $\mathrm{NaCl}$ en la solución de la pectina del níspero de la sierra al $\mathbf{0 , 5 \%}$

\begin{tabular}{cccc}
\hline Muestra & Concentración de NaCl (M) & Tiempo (minutos) & $\boldsymbol{\eta}_{\text {relativa }}$ \\
\hline 1 & 0,0 & 229 & 2,90 \\
2 & 0,2 & 120 & 1,70 \\
3 & 0,4 & 135 & 1,79 \\
4 & 0,6 & 144 & 1,86 \\
5 & 0,8 & 144 & 1,86 \\
\hline
\end{tabular}

\section{Gráfica 7}

Efecto del $\mathrm{NaCl}$ en la solución de la pectina del níspero de la sierra al $0,5 \%$

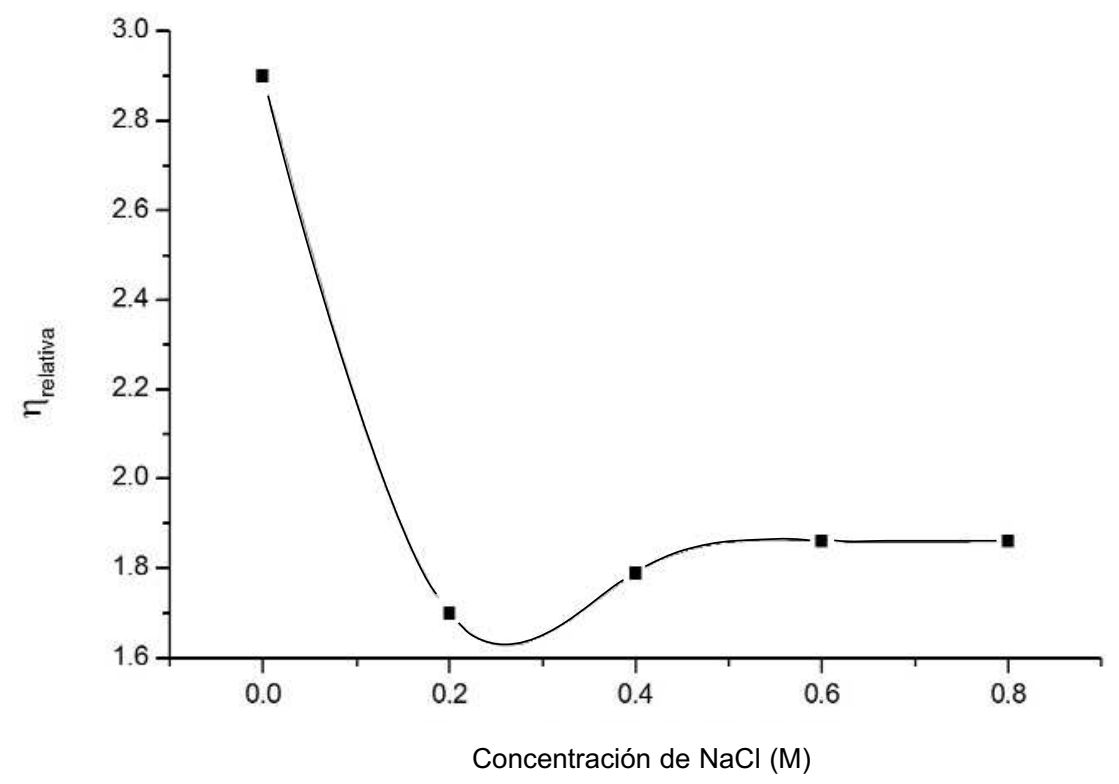

En la gráfica 7 se observa que la solución de la pectina del níspero de la sierra forma geles a concentraciones entre 0,2 y $0,8 \mathrm{M}$ de $\mathrm{NaCl}$ a partir de un lapso de 2 minutos. 
La tabla 5 y la gráfica 8 muestran los resultados obtenidos al añadir $\mathrm{CaCl}_{2}$ a la solución de la pectina del níspero de la sierra al 0,5\%.

Tabla 5

Influencia del $\mathrm{CaCl} 2$ en la solución de la pectina del níspero de la sierra al $0,5 \%$

\begin{tabular}{cccc}
\hline Muestra & Concentración de $\mathbf{C a C l}_{\mathbf{2}}$ (mM) & Tiempo (segundos) & $\mathbf{\eta}_{\text {relativa }}$ \\
\hline 1 & 0 & 229 & 2,90 \\
2 & 1 & 299 & 3,81 \\
3 & 2 & 320 & 4,10 \\
4 & 3 & 343 & 4,24 \\
5 & 4 & 420 & 4,32 \\
6 & 5 & 535 & 4,40 \\
\hline
\end{tabular}

Gráfica 8

Efecto del $\mathrm{CaCl}_{2}(\mathrm{mM})$ en la pectina del níspero de la sierra al $0,5 \%$

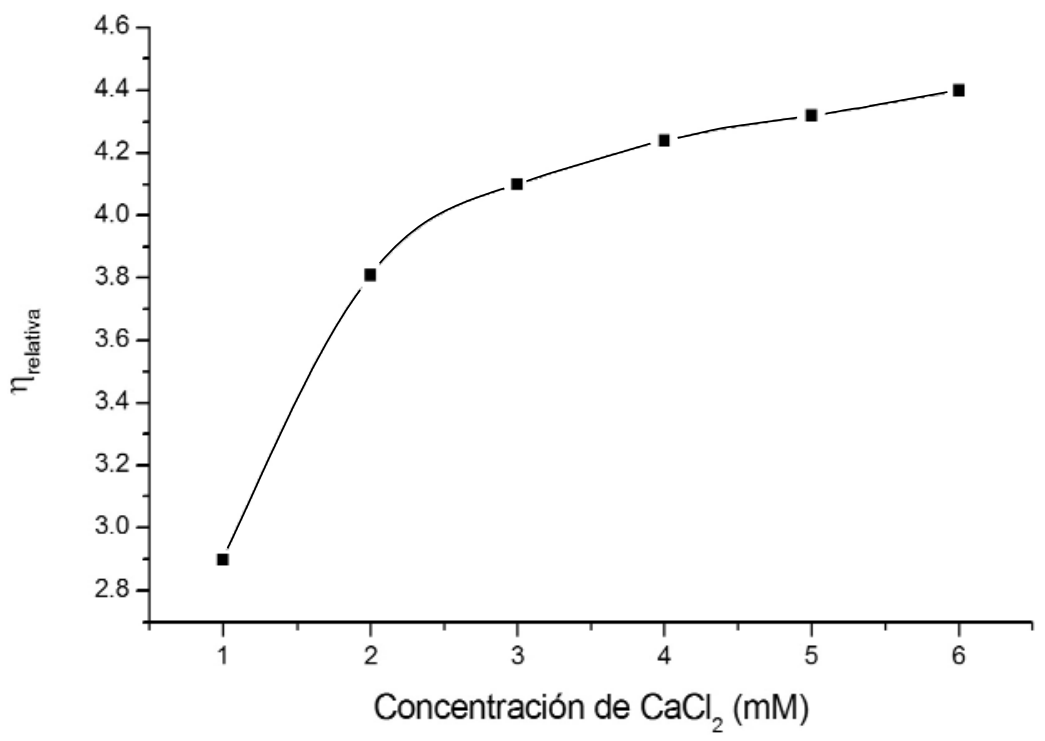


En la gráfica 8 se observa que la adición de pequeñas cantidades de calcio promovieron significativamente la gelación de la solución de la pectina del níspero de la sierra. Adicionalmente, el incremento de la concentración de calcio por encima de $5 \mathrm{mM}$ no cambia la fuerza del gel (Singthong et al. 2005: 793-801). La gelación de la muestra también se ve favorecida por el contenido de $\mathrm{Ca}^{+2}$ que presenta la muestra (tabla 1).

La tabla 6 y la gráfica 9 muestran los resultados que se obtienen al añadir sacarosa a la solución de la pectina del níspero de la sierra al $0,5 \%$.

\section{Tabla 6}

Efecto de la sacarosa en la solución de la pectina del níspero de la sierra al $0,5 \%$

\begin{tabular}{cccc}
\hline Muestra Concentración de sacarosa (M) & Tiempo (segundos) & $\boldsymbol{\eta}_{\text {relativa }}$ \\
\hline 1 & 0,0 & 229 & 2,90 \\
2 & 0,2 & 299 & 3,81 \\
3 & 0,4 & 320 & 4,32 \\
4 & 0,6 & 343 & 4,51 \\
5 & 0,8 & 458 & 6,30 \\
6 & 1,0 & 535 & 7,10 \\
\hline
\end{tabular}

Gráfica 9

Efecto de la sacarosa en la solución de la pectina del níspero de la sierra al $0,5 \%$

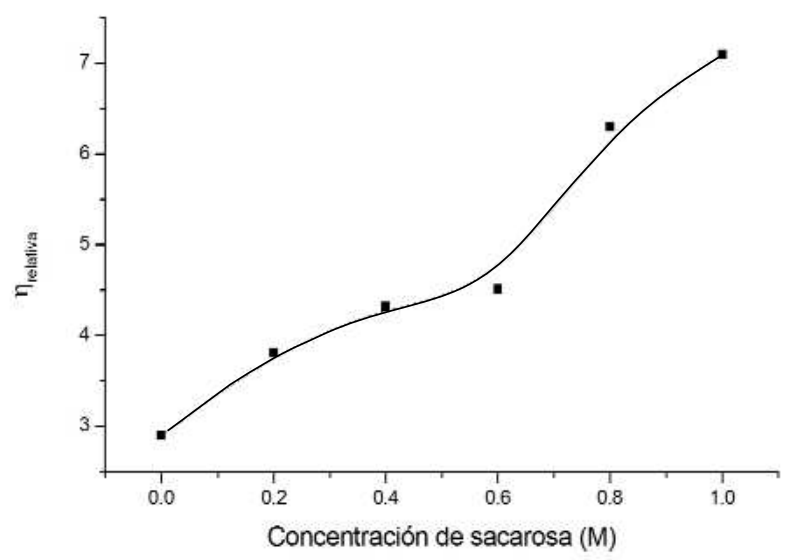


En la gráfica 9 se observa que la fuerza del gel de la solución de la pectina del níspero de la sierra al $0,5 \%$ es incrementada por la sacarosa, existiendo una correlación directa entre la concentración de la sacarosa y la fuerza del gel. La sacarosa incrementa la viscosidad reducida de la pectina y este efecto aumenta con el incremento de la concentración de la sacarosa por encima de 0,6 M (Singthong et al. 2005: 793-801).

\section{CONCLUSIONES}

- La gelación de la pectina del níspero de la sierra ocurre bajo condiciones alcalinas en presencia de $\mathrm{NaOH}$. Se observó que el gel comienza a formarse a los dos minutos de adicionado el $\mathrm{NaOH}$. Este resultado indica que las condiciones alcalinas conducen a la desmetoxilación de la pectina y asegura la disociación de los grupos carboxílicos.

- Del análisis de la densidad relativa respecto a la concentración de la pectina del níspero de la sierra se concluye que el gel se forma a concentraciones mayores de entre 1,5 y $2,5 \%$.

- Del estudio de la densidad relativa respecto al pH del medio se concluye que a $\mathrm{pH} 5$ la molécula de la pectina del níspero de la sierra se encuentra enrollada, por lo que presenta una baja viscosidad relativa.

- Los co-solutos ayudan en la formación del gel del níspero de la sierra, siendo mucho mejor la influencia del ion calcio. Además, se observó que la adición de sacarosa incrementa la fuerza del gel.

- Se concluye que la pectina extraída del níspero de la sierra tiene un gran potencial como un hidrocoloide comercial por presentar propiedades de gelación únicas, por lo que puede ser empleado en la manufactura de mermeladas, jaleas, confites, yogur de frutas, bebidas de bajas calorías, alimentos probióticos, y productos farmacéuticos y dietéticos, ya que tienen un efecto positivo en la reducción de colesterol en la sangre.

- Esta investigación contribuye a incrementar el valor agregado de frutos autóctonos y a la creación de nuevos productos para el mercado interno y la agroexportación. 


\section{BIBLIOGRAFÍA}

Arévalo, R. P.; Quast, L. B. y T. G. Kieckbusch (2006). Viscosidad aparente de la pulpa del camu camu (Myrciaria dubia) in natura y con pretratamiento térmico. Buenos Aires: Asociación Argentina de Ingenieros Químicos.

Axelos, M. A. V. y J. F. Thibault (1991). "The chemistry of low metoxyl pectin gelation", en Walter, R. H.. The chemistry and technology of pectin. Nueva York: Academic Press.

Barbosa-Canovas, G. V; Ibarz, A. y M. Peleg (1993). "Propiedades reológicas de alimentos fluidos". Revisión Alimentaria 241.

Barford, R. A. et al. (1986). "Estimation of degree of methylation of pectin by pyrolysis-gas chromatography". Analytical Chemistry 58.

Bernhard, W. J.; Menzies, N. W. y F. P. C. Blamey (2004). "Alkali hydroxide-induce gelation of pectin". Food Hydrocolloids 18.

Chasquibol S., N.; Arroyo B., E. y J. C. Morales (2008). "Extracción y caracterización de pectinas obtenidas a partir de frutos de la biodiversidad peruana”. Ingeniería Industrial 26. Lima: Universidad de Lima, Fondo Editorial.

Choi, Y. H. y B. Yoo (2004). "Characterization of time-dependent flow properties of food suspensions". International Journal of food Science and Technology V, 39.

Goycoolea F. M. y A. Cárdenas (2003). "Pectins from Oppuntia spp: A short review". J. PACD.

Grant, G. T. et al. (1973). "Biological interactions between polysaccharides and divalent cations: The egg-box model". FEBS Letters 32.

Gregory, D. J. H. (1986). "Disfunctional properties of pectincs in various food systems", en Birch, G. G. y M. G. Lindley (eds.). Interactions of food components. Londres: Elsevier.

Koseki, M. et al. (1986). "Determination de pectin in the presence of food polysaccharides". Journal of Food Science 51 (5).

Manrique G. D. y F. M. Lajolo (2002). "FT-IR spectroscopy as a tool for measuring degree of methyl esterification in pectins isolated from ripening papaya fruit”. Postharvest Biology and Technology 25. 
May, D. C. "Industrial pectins: Sources, production and applications". Carbohydrate Polymers 12.

Medina-Torres, L. et al. (2002). "Rheological properties of the mucilage gum (Opuntia picus indica)". Food Hydrocolloids 14.

Oakenfull, D. G. y A. Scott (1984). "Hydrophobic interaction in the gelation of high metoxyl pectins". Journal of Food Science 49 (4).

Oakenfull, D. G. (1991). "The chemistry of high-metoxyl pectin gelation", en Walter, R. H. The chemistry and technology of pectin. Nueva York: Academic Press.

Phatak, L.; Chang, C. K. y G. Brown (1988). "Isolations of characterization of pectin in sugar-beet pulp". Journal of Food Science 53 (3).

Rao, M. A. (1986). Engeneering properties of foods. Nueva York: Basel (Ed) Marcel Dekker.

Singthong, J. et al. (2005). "Extraction and physicochemical characterization of Krueo Ma Noy pectin”. Food Hydrocolloids 19.

Vidal, J. R. M. B.; Gasparetto, C. A. y A. Grandin (2000). "A efeito da temepratura no comportamento reológico de poupa de manga". Revista de Ciencias Exatas e Naturais 70.

Voyutsky, S. (1978). Colloid chemistry. Moscú: MIR Publishers. 